\title{
Deep Learning Analysis: A Review
}

\author{
P. Rajendra Kumar ${ }^{1}$, Suban Ravichandran ${ }^{2}$ and Narayana Satyala ${ }^{3}$ \\ ${ }^{1}$ Research Scholar, Department of Computer Science and Engineering \\ ${ }^{2}$ Assistant Professor, Department of Information Technology, Faculty of Engineering and Technology, \\ ${ }^{1 \& 2}$ Annamalai University, Annamalai Nagar, Tamil Nadu, India \\ ${ }^{3}$ Professor \& Head of the Department, Department of Computer Science and Engineering \\ Gudlavalleru Engineering College, Gudlavalleru, Andhra Pradesh, India \\ E-Mail: p.rajendrakumar08@gmail.com, rsuban82@gmail.com, Satyala1976@gmail.com
}

\begin{abstract}
Deep learning is a rising territory of machine learning (ML) inquires about. It includes different shrouded layers of fake neural systems. Deep learning (DL) is a part of machine learning dependent on an arrangement of calculations that endeavor to show abnormal state reflections in information. It is utilized by Google in its voice and picture acknowledgment calculations, by Netflix and Amazon to choose what you need to watch or purchase straightaway, and by specialists at MIT to anticipate what's to come. Profound Learning is utilized in different fields for accomplishing various levels of deliberation like sound, content; pictures highlight extraction and so forth. The Deep learning philosophy applies nonlinear changes and model reflections of abnormal state in extensive databases. With Deep learning capacity to make forecasts and groupings taking the upside of huge information, it can be a creative answer for issues and issues that have been never thought to be understood in such a simple way. Then again, it makes numerous difficulties on the researchers who are endeavoring to convey such another methodology. The accompanying audit sequentially shows how and in what real applications profound realizing calculations have been used. We have completed a broad writing audit and reviewed the utilization of profound learning methods on different fields.
\end{abstract}

Keywords: Deep Learning, Machine Learning, Reinforcement Learning, Speech Recognition, and Image Processing

\section{INTRODUCTION}

The execution of machine learning techniques is vigorously reliant on the selection of information portrayal on which they are connected [1]. Therefore, a great part of the genuine exertion in sending machine learning calculations [2] goes into the structure of preprocessing pipelines and information changes that outcome in a portrayal of the information that can bolster powerful machine learning. Such component designing is imperative yet work concentrated and features the shortcoming of current learning calculations: their failure to separate and sort out the discriminative data from the information. Highlight designing [4] is an approach to exploit human resourcefulness and earlier information to make up for that shortcoming. The most exemplary subdivision inside NLP is machine interpretation, or, in other words the interpretation between dialects. Machine interpretation calculations have brought about different applications that consider syntax structure and spelling botches. Also, an arrangement of words and vocabulary identified with the primary theme is consequently utilized as the principle source when the PC is proposing changes to essayist or manager [3]. With the end goal to grow the extension and simplicity of materialness of machine learning, it would be very attractive to make learning calculations less subject to highlight designing, so novel applications could be developed quicker, and all the more essentially, to gain ground towards Artificial Intelligence (AI). Measurable bends can depict past, and present with the end goal to foresee future practices. In any case, amid the most recent decades just great procedures and calculations have been utilized to process this information, while an enhancement of those calculations could lead on a viable self- learning [17]. A superior basic leadership can be actualized dependent on existing qualities, numerous criteria and insights propelled techniques.

A. Types of Deep Machine Learning: Prior to getting into the papers, allows first discuss what fortification realizing is. This field of Machine Learning can be isolated into three principle classes shown in Fig.1.

1. Supervised learning.

2. Unsupervised learning.

3. Reinforcement learning.

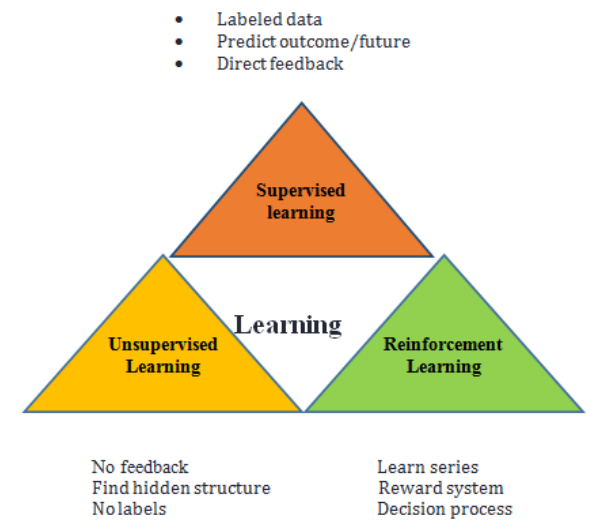

Fig.1 Different types of Learning

\section{Supervised Learning}

Principal classification, supervised learning, is the one you 
might be most acquainted with. It depends on making a capacity or model dependent on an arrangement of preparing information, which contains inputs and their comparing names. Convolutional neural systems are an extraordinary case of this, as the pictures are the information sources and the yields are the arrangements of the pictures (pooch, feline, and so on.).

\section{Unsupervised Learning}

Unsupervised learning tries to discover a type of structure inside information through techniques for bunch examination. A standout amongst the most outstanding ML bunching calculations, k-implies, is a case of unsupervised learning.

\section{Reinforcement Learning}

Reinforcement learning is the undertaking of realizing what moves to make, given a specific circumstance or condition, in order to amplify a reward flag. The intriguing distinction among administered and support learning is that this reward flag basically discloses to you whether the activity (or information) that the operator takes is great or terrible. It doesn't disclose to you anything about what the best activity is. Difference this to CNNs, where the comparing name for each picture input is a distinct guidance of what the yield ought to be for each info.

Another remarkable part of $\mathrm{RL}$ is that an operator's activities will influence the ensuing information it gets. For instance, a specialist's activity of moving left rather than right implies that the operator will get diverse contribution from the earth at whenever step. We should take a gander at a precedent to begin off.

\section{B. The Reinforcement Learning Problem}

Along these lines, allows first consider what have in a fortification learning issue. How about we envision a little robot in a little room. We haven't modified this robot to move or walk or make any move. It's simply remaining there. This robot is our operator.

Like we specified previously, support learning is tied in with endeavoring to comprehend the ideal method for deciding/activities, so we expand some reward(R). This reward is a criticism flag that just demonstrates how well the operator is getting along at a given time step.

The activity (A) that an operator makes at each time stride is an element of both the reward (flag telling the specialist how well it's at present doing) and the state (S), or, in other words of the condition the specialist is in.

The mapping from condition states to activities is called our arrangement $(\mathrm{P})$. The strategy essentially characterizes the operator's method for acting at a specific time, given a specific circumstance.

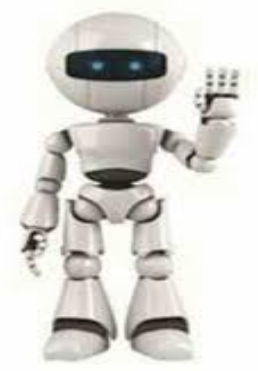

Fig. 2Artificial intelligence/Robot Apocalypse

Presently, we additionally have an esteem work (V), or, in other words of how great each position is. This is not the same as the reward in that the reward flag demonstrates what is great in the quick sense, while the esteem work is more characteristic of the fact that it is so great to be in this state/position over the long haul. At long last, we likewise have a model (M), or, in other words portrayal of the earth. This is the specialist's model of how it imagines that nature will carry on.

\section{Biomedical Data in Deep Learning}

\section{Managing Variable-Size Structured Data:}

Feed forward neural systems are routinely used to process input vectors or tensors of settled size. For example, in a PC vision application, every one of the pictures can be seen as settled size frameworks. Be that as it may, in numerous biomedical applications, the information arrives in an alternate arrangement, where information things shift in size and are frequently connected with a fundamental graphical structure. This is the situation, for instance, of little particles, nucleotide or amino corrosive groupings, protein or other contact maps, phylogenetic trees, characteristic dialect arrangements, and common dialect parse trees. In a considerable lot of these cases, the extent of the information things can change incredibly, and utilizing a feedforward arrange tuned to the maximal conceivable size is generally not a decent methodology. In this way, in these cases, a recursive system must be utilized. A recursive neural system is a neural system comprising of submodules that happen over and over. An intermittent system unfurled in time is a case of a straightforward recursive system. Recursive neural system methodologies can likewise be seen as messagepassing calculations, where factors related with hubs or edges of a chart are refreshed as a component of the factors present in their diagram neighborhood. The refresh capacities are executed by neural systems and these systems can be partaken in space or time. All the more solidly, when planning recursive neural systems to process variableestimate organized information, it is valuable to recognize two essential methodologies: the internal methodology and the external methodology (13).

\section{Inward and Outer Recursive Neural Network Approaches}

In the internal methodology, the information show must be caught by a coordinated non-cyclic chart, and neural 
systems are utilized to figure a vector at every hub of the diagram as a component of the vectors related with the parent hubs. In the event that the diagram has a customary structure, for instance, similar to a chain, matrix, or some other grid, at that point the systems related with comparative hubs can be shared subsequently the recursive angle. For instance, on account of groupings, these can be demonstrated utilizing Bayesian systems related with coordinated acrylic charts. For this situation, the internal methodology utilizes two recursive neural systems, one for the changes and one for the discharges. The methodology is called internal in light of the fact that the neural systems are utilized to slither the charts related with the information from within. For successions, it is conceivable to creep them in the two bearings, from the beginning to the end and the other way around, and this prompt bidirectional recursive neural systems(BRNNs).

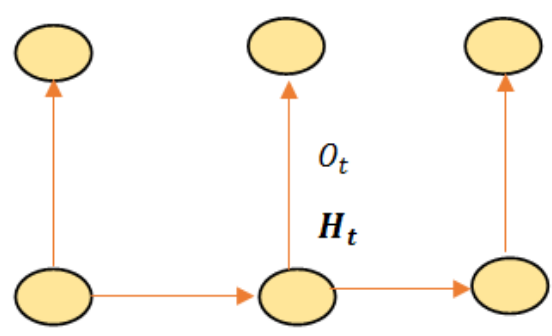

Fig.3. Recursive neural network inner sequence containing hidden Markov model, hidden state $\mathrm{H}_{\mathrm{t}}$ \& Output $\mathrm{O}_{\mathrm{t}}$ at time t.

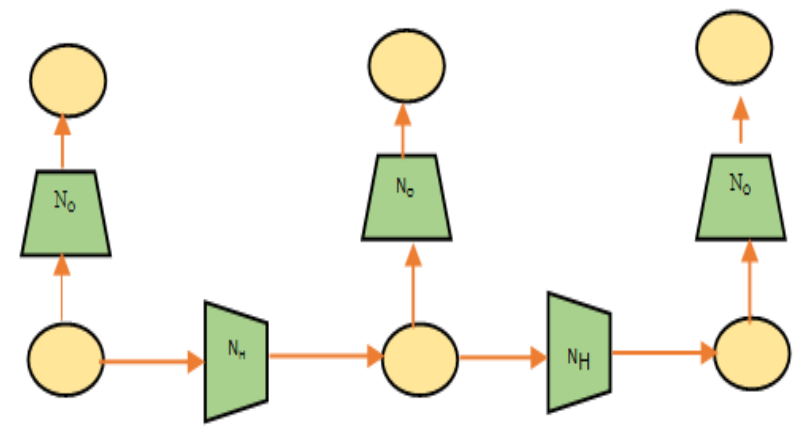

Fig. 4 Recursive neural network inner sequence containing two separate networks, one to computer hidden state \& output positions and back propagation

\section{APPLICATIONS}

AI, machine learning, and deep learning have gained a lot of attention for quite some time now. Deep Learning is changing the manner in which we take a gander at advances. There is a ton of energy around Artificial Intelligence (AI) alongside its branches in particular Machine Learning (ML) and Deep Learning right now. It's anticipated that numerous Deep learning applications will influence your life sooner rather than later. As a matter of fact, they are now having an effect. Inside the following 5 to 10 years, Deep learning advancement instruments, libraries, and dialects will end up standard parts of each product improvement toolbox. Deep learning can be comprehended as a strategy to enhance results and improve handling times in a few figuring forms. In the field of common dialect preparing, profound learning techniques have been connected for picture inscription age [5], and handwriting generation [6]. These technologies are revolutionizing various industries such as retail, finance, manufacturing, healthcare, Medicine and etc.

\section{A. Deep Learning in Image Processing}

Another prevalent region with respect to deep learning is picture acknowledgment. It intends to perceive and recognize individuals and protests in pictures and additionally to comprehend the substance and setting. Picture acknowledgment is now being utilized in a few segments like gaming, internet-based life, retail, tourism, and so on. Before deep adapting formally showed up as another exploration approach, a few applications had been done inside the idea of example acknowledgment through layer handling. In 2003, an intriguing precedent was created by applying molecule sifting and Bayesian - conviction proliferation. The principle idea of this application recommends that a human can perceive the essence of a man by observing just a half - edited face picture [7], along these lines a PC might recreate the picture of a face from a trimmed one. Later in 2006, voracity calculation and order were consolidated into an application able to process written by hand digits [8]. Ongoing examines have connected deep learning as the primary apparatus for computerized picture handling. For example, applying a Convolutional Neural Networks (CNN) for Iris Recognition can be more compelling than utilizing ordinary iris sensors. CNN adequacy can reach up to $99.35 \%$ of exactness [9]. Portable area acknowledgment these days enables the client to know a decided location dependent on an image. A Supervised Semantics - Preserving Deep Hashing (SSPDH) calculation has demonstrated an extensive enhancement in correlation with Visual Hash Bit. This undertaking requires the order of articles inside a photo as one of an arrangement of beforehand known items. A more perplexing variety of this undertaking called question location includes particularly distinguishing at least one protest inside the scene of the photo and drawing a container around them.

\section{B. DL in Speech Recognition or Voice Search \& Voice- Activated Assistants}

One of the most popular usage areas of deep learning is voice search \& voice-activated intelligent assistants. With the enormous tech monsters have officially made critical interests around there, voice-initiated partners can be found on almost every cell phone. Apple's Siri is available since October 2011. Google Now, the voice-enacted right hand for Android, was propelled not as much as a year after Siri. The most up to date of the voice-actuated savvy associates is Microsoft Cortana.

Li Deng and John C[10] Platt review introduces that deep learning frameworks have drastically enhanced the exactness 
of discourse acknowledgment, and different deep structures and learning strategies have been produced with particular strengths $[11,12]$ and shortcomings as of late. Deep learning is ordinarily connected to PC vision, discourse acknowledgment, and NLP. These are non-straight characterization problems where the data sources are very hierarchal in nature.

In 2011, Google Brain venture, made a neural system prepared with deep learning calculations, which perceived abnormal state ideas, similar to felines, in the wake of observing just YouTube recordings and without being told what a "feline" is. Facebook [14] is making arrangements utilizing profound learning aptitude to all the more likely recognize faces and protests in the photographs and recordings transferred to Facebook each day $[15,16,17]$. Another case of deep learning in real life is voice acknowledgment like Google Now and Apple's Siri. As indicated by Google, the voice blunder rate in the new form of Android remains at $25 \%$ lower than past renditions of the product in the wake of including experiences from deep learning.

\section{Predicting Earthquakes}

Harvard researchers utilized Deep Learning to show a PC to perform viscoelastic calculations, these are the calculations utilized in expectations of seismic tremors. Until their paper, such calculations were exceptionally PC concentrated, yet this utilization of Deep Learning enhanced figuring time by $50,000 \%$. With regards to tremor estimation, timing is imperative, and this enhancement can be fundamental in sparing a real existence.

\section{Neural Networks in Finance}

Prospects markets have seen an extraordinary accomplishment since their commencement both in created and creating nations amid the most recent four decades. This achievement is inferable from the huge use the fates give to showcase members. This examination dissects an exchanging methodology which profits by this use by utilizing the Capital Asset Pricing Model (CAPM) and cost-of-convey relationship. The group applies the specialized exchanging rules created from spot showcase costs, on prospects advertise costs utilizing a CAPM based support proportion. Verifiable day by day costs of twenty stocks from every one of the ten markets (five created markets and five developing markets) are utilized for the examination.

\section{E. Advertising}

Promoting is another key region that has been changed by profound learning. It has been utilized by the two distributers and sponsors to build the importance of their advertisements and lift the arrival on venture of their publicizing efforts. For example, deep learning makes it feasible for promotion systems and distributers to use their substance with the end goal to make information driven prescient publicizing, constant offering real-time bidding(RTB) for their advertisements, accurately focused in plain view publicizing and that's just the beginning.

\section{F. Medicine}

Computerized picture handling is certainly a critical piece of the examination fields where a deep learning strategy can be connected. In this way, clinical applications have been as of late tried. For example, a correlation between shallow learning and deep learning in neural systems leaded to a superior execution on illness expectation. A picture taken from a Magnetic Resonance Imaging (MRI) [19] from a human cerebrum was handled with the end goal to anticipate a conceivable Alzheimer sickness [18]. Despite the fact that the early accomplishment of this strategy, a few issues ought to be considered for future applications. Preparing and reliance on high caliber is a portion of the confinements. Volume, quality and intricacy of information are testing viewpoints, anyway the coordination of heterogeneous information composes is a potential part of profound learning design [20]. Optical Coherence Tomography (OCT) is another precedent where deep learning strategies are demonstrating profitable outcomes. Customarily, pictures are treated by a manual improvement of convolution frameworks. Shockingly, the absence of preparing sets restricts the deep learning technique. By the by inside a couple of years, the presentation of better preparing sets will successfully foresee retinal pathologies and decline OCT innovation costs.

\section{CONCLUSION}

Deep learning is undoubtedly a quickly developing use of machine learning. The various applications portrayed above demonstrate its quick improvement in only couple of years. The use of these calculations in various fields demonstrates its adaptability. The distribution investigation performed in this examination unmistakably exhibits the importance of this innovation and gives an obviously outlines the development of profound learning and the propensity with respect to for future research in this field. Moreover, take note of that progressive system of layers and the supervision in learning are key variables to build up a fruitful application with respect to profound learning. Deep learning is simply extremely gigantic fake neural systems equipped for utilizing considerably more volumes of information to learn with expanding execution. The field is developing rapidly, with new structures and learning strategies exhibited always. The once-over of unsupervised models we have investigated in this segment is assuredly not exhaustive. In the midst of the latest couple of years, different new profound learning models for unsupervised learning have been proposed. Scientist starting late proposed a to some degree specific profound learning-based structure for unsupervised learning, called generative stochastic framework which intends to take in a Markov chain Monte Carlo move head instead of a full probability dissemination. 


\section{REFERENCES}

[1] O. Abdel, "Applying convolutional neural networks concepts to hybrid NN-HMM model for speech recognition”, Acoustics, Speech and Signal Processing, Vol. 7, pp. 4277-4280, 2012.

[2] A. Mosavi and A. Varkonyi-Koczy, "Integration of machine learning and optimization for robot learning", Advances in Intelligent Systems and Computing, Vol. 519, pp. 349-355, 2017.

[3] A. Mosavi, A. Varkonyi-Koczy and M. Fullsack, "Combination of machine learning and optimization for automated decision-Making”, in International Conference on Multiple Criteria Decision Making, 2015.

[4] A. Coates,H. Lee and Y. Ng. Andrew, "An analysis of single- layer networks in unsupervised feature learning", in International Conference on Artificial Intelligence and Statistics, 2011.

[5] Li. Deng and Yu. Dong, "Deep learning: methods and applications", Foundations and Trends ${ }^{\circledR}$ in Signal Processing, Vol. 7, No. 3-4, pp.197-387, 2014.

[6] A. Mohamed, "Deep belief networks for phone recognition”, Nips Workshop on Deep Learning for Speech Recognition and Related Applications, Vol. 1, pp. 635-645, 2009.

[7] A. Mosavi and A. Vaezipour, "Reactive search optimization; application to multi objective optimization problems”, Applied Mathematics, Vol. 3, pp.1572-1582, 2012.

[8] B. Goel, "Developments in the field of natural language processing", International Journal of Advanced Research in Computer Science, Vol. 8, No. 3, pp. 23-28, 2017.

[9] T.S. Lee and D. Mumford, "Hierarchical Bayesian inference in the visual cortex", in Journal of the Optical Society of America, Vol. 20, No. 7,pp.1434-1448, 2003.
[10] Y. Le Cun, Y. Bengio and Geoffrey Hinton, “Deep learning”, Nature, pp. 436-444, 2015.

[11] A. Rushton, “Formative assessment: a key to deep learning?” 2007.

[12] P. Kiran Sree, "A fast multiple attractor cellular automata with modified clonal classifier for coding region prediction in human genome”, Journal of Bioinformatics and Intelligent Control, Vol. 3, pp.1-6, 2014.

[13] P. Baldi, “Deep Learning in Biomedical Data Science”, 2018.

[14] P. KiranSree, "Investigating an artificial immune system to strengthen the protein structure prediction and protein coding region identification using cellular automata classifier”, International Journal of Bioinformatics Research and Applications ,Vol. 5, No. 6,pp. 647-662, 2009.

[15] P.KiranSree, "Identification of promoter region in genomic DNA using cellular automata based text clustering”, The International Arab Journal of Information Technology, Vol.7, No.1, pp.75-78, 2010.

[16] David H. Hubel and Torsten N. Wiesel, "Receptive fields, binocular interaction and functional architecture in the cat's visual cortex", in Journal of Physiology, Vol. 160, No. 1, pp.106-154, 1962.

[17] S. Safdar, S. Zafar, N. Zafar and N.F. Khan, "Machine learning based decision support systems (DSS) for heart disease diagnosis: a review”, Artificial Intelligence Review, pp. 1-17, 2017.

[18] Vaezipour, A. Mosavi and U. Seigerroth, "Visual analytics and informed decisions in health and life sciences", in International CAE Conference, Verona, Italy, 2013.

[19] W. Liu, "Deep learning hashing for mobile visual search", in EURASIP Journal on Image and Video Processing, 2017.

[20] R. Salakhutdinov and Geoffrey Hinton, "Deep boltzmann machines”, in Artificial Intelligence and Statistics, pp.448-455, 2009. 